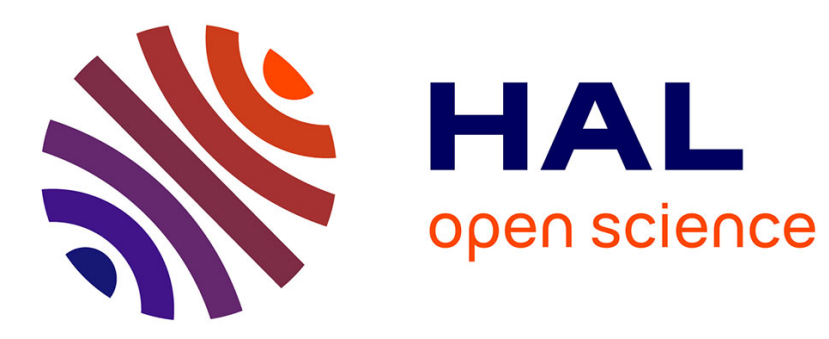

\title{
Spectral fluctuation in SERS spectra of benzodiazepin molecules: The case of oxazepam
}

Alexandre Merlen, Cédric Pardanaud, Katharina Gratzer, Stéphane Coussan, Denis Machon, Alexis Forestier, David Bergé-Lefranc, Trang Phan, Virginie Hornebecq

\section{To cite this version:}

Alexandre Merlen, Cédric Pardanaud, Katharina Gratzer, Stéphane Coussan, Denis Machon, et al.. Spectral fluctuation in SERS spectra of benzodiazepin molecules: The case of oxazepam. Journal of Raman Spectroscopy, 2020, 51 (11), pp.2192-2198. 10.1002/jrs.5972 . hal-02928096

\section{HAL Id: hal-02928096 https://hal.science/hal-02928096}

Submitted on 24 Feb 2021

HAL is a multi-disciplinary open access archive for the deposit and dissemination of scientific research documents, whether they are published or not. The documents may come from teaching and research institutions in France or abroad, or from public or private research centers.
L'archive ouverte pluridisciplinaire HAL, est destinée au dépôt et à la diffusion de documents scientifiques de niveau recherche, publiés ou non, émanant des établissements d'enseignement et de recherche français ou étrangers, des laboratoires publics ou privés. 
Spectral fluctuation in SERS spectra of benzodiazepin molecules: the case of oxazepam

A. Merlen*1, C. Pardanaud*2, K. Gratzer ${ }^{1,2}$, S. Coussan' ${ }^{2}$, D. Machon ${ }^{3}$, A. Forestier ${ }^{3}$, D. Bergé-Lefranc ${ }^{4}$, T. N. T. Phan ${ }^{5}$ and V. Hornebecq ${ }^{6}$

1 Univ Toulon and Aix-Marseille Univ, CNRS, IM2NP, UMR 7334, site de Toulon, France

2 Aix-Marseille Univ, CNRS, PIIM, Marseille, France.

3 Univ Lyon, Université Claude Bernard Lyon 1, CNRS UMR 5306, Institut Lumière Matière, F-69622 Villeurbanne, France

4 Aix Marseille Univ, CNRS, IMBE, Faculté de Pharmacie, Marseille, France

5 Aix Marseille Univ, CNRS, Institut de Chimie Radicalaire, Marseille, France

6 Aix Marseille Univ, CNRS, MADIREL, Marseille, France

merlen@univ-tln.fr

cedric.pardanaud@univ-amu.fr

\section{$\underline{\text { Abstract }}$}

The SERS spectra of a benzodiazepine molecule, oxazepam, have been recorded using a nanoporous silica matrix containing gold nanoparticles as SERS substrate. In the measured spectra, we clearly observe spectral fluctuations, with slight shifts in wavenumber, appearance and disappearance of peaks and variations in the shape of some peaks. By comparison with spectra simulated by Density Functional Theroy (DFT), we attribute those fluctuations to the experimental signature of various adsorption sites of the molecule on the gold surface. The effect of temperature and laser power was also studied, suggesting that changes in absorption sites can be optically activated. Such features could cause misinterpretation of SERS spectra and errors in the identification of drugs. We suggest that they should be systematically considered for the correct interpretation of SERS spectra of drug molecules.

\section{$\underline{\text { Introduction }}$}

Drug abuse remains a serious public health problem all around the world and the rapid and efficient detection of drugs in blood or other body fluids remains a challenge. Among all the techniques in development, Surface Enhanced Raman Spectroscopy (SERS) appears as highly promising with respect to selectivity and sensitivity ${ }^{[1-3]}$. Indeed, standard Raman Spectroscopy has already proven to be efficient for drugs identification ${ }^{[4]}$ but it suffers a major limitation: its very low cross section. In this way, this technic can be used for the analysis of bulk samples but in blood or saliva, the concentration of drugs is usually too low for an efficient and direct analysis based on classical Raman spectroscopy. As an alternative, SERS combines the chemical selectivity of Raman spectroscopy associated to a very high sensitivity, as single molecule detection has already been proven with this method ${ }^{[5]}$. It is based on the gigantic electromagnetic enhancement obtained with plasmonic nano-antennas ${ }^{[6]}$. This enhancement occurs in the near field of the nano-antenna, a few nanometers above its surface. In most SERS studies, those nano-antennas are either gold or silver nanoparticles. Usually gold is privileged due to its better chemical stability and lower toxicity compared to silver, even if this last 
point remains subject of debate ${ }^{[7]}$. The experimental protocol for performing SERS is straightforward: the solution is deposited on a SERS substrate, usually made of gold nanoparticles or nanostructures, and the spectrum is directly recorded via a standard Raman spectrometer. In some cases, the measurement can be directly performed in a solution containing the nano-antennas. Thus, except the elaboration of nano-antennas, SERS does not require any specific experimental device and can be easily performed. Since its development in the 70's, many methods have been developed for the preparation of efficient SERS substrates, with a large variety of shape for the nano-antennas ${ }^{[8]}$ : spheres, triangles, pyramids, stars etc......Nevertheless the preparation of efficient nano-antennas is not the only key point in SERS. Another important point is the nature of the chemical interaction between the metallic nanostructures and the probe molecules, this feature is generally called the "chemical enhancement" of SERS ${ }^{[9]}$. It has led to an extensive debate in the SERS community but it is generally admitted that it is related to a charge transfer mechanism between the metal and the molecule ${ }^{[10]}$. Its relative contribution to the final enhancement of the Raman signal remains unclear as it varies depending on the combination of sample and antenna, however, either way, this feature underlines that the interaction between the nano-antenna and the molecules must be taken into account for the interpretation of SERS spectra.

In the specific case of benzodiazepine, SERS has already proven to be efficient ${ }^{[11-13]}$. Those pharmacological molecules are used as tranquilizers and sleep-inducing agents in particular. Doctors can prescribe a benzodiazepine for legitimate medical conditions but any abuse can create addiction and have severe health consequences. The detection of those molecules is a challenge and SERS is a promising alternative to other established techniques. The basic principle of benzodiazepine detection is based on a spectral analysis by identification of characteristic vibrational modes. The main problem with this approach is that previous SERS studies of benzodiazepine have reported differences between the powder Raman spectrum of those molecules and the observed SERS spectra. The authors have suggested that those differences should be a sign of the interaction of the molecule with the metallic surface without giving too much detail. In order to address reproducibility and biases introduced by measurements, it is necessary to understand the origin of those spectral differences. Any changes in the spectral fingerprint of a molecule should be clearly identified and understood for the further development of SERS as a reliable detection technique. Here, comparing SERS measurements and DFT calculations, an explanation for the spectral fluctuations of SERS spectra of oxazepam, a benzodiazepine molecule, is proposed.

\section{Experimental and methods}

SERS samples are based on nanoporous silica matrix containing gold nanoparticles in its pores $\left(d_{\text {pore }}\right.$ is close to 8-9 $\mathrm{nm})^{[14]}$. They were prepared following the procedure described in ${ }^{[15]}$. Transmission Electron Microscopy observations and low wavenumber Raman measurement (below $10 \mathrm{~cm}^{-1}$ ) confirmed the presence of gold nanoparticles in the silica matrix with typical sizes close to $5 \mathrm{~nm}$ (see supplementary information). With such small nanoparticles, the crystallinity of gold is very poor and their surfaces should exhibit a large variety of various adsorption sites for oxazepam molecules. Oxazepam was adsorbed on the material surface using a classical procedure. A known mass of solid is put in contact with an oxazepam solution of known concentration. After reaching equilibrium (12 hours), the supernatant is recovered and the equilibrium oxazepam concentration is determined. In this way, it is possible to calculate the adsorbed amount of oxazepam per gram of solid. In this study, this value is close to $2 \mu \mathrm{mol} / \mathrm{g}$. All the results obtained in this study were obtained with dried samples consisting of a red powder. This color is due to the gold nanoparticles. 
SERS measurements were performed in backscattering geometry on grains (zones of analysis were selected to be as flat as possible to optimize signal intensity) using $633 \mathrm{~nm}$ laser excitation with a LabRam HR800 spectrometer from the Horiba Jobin Yvon Company. It must be mentioned that we have also used a $514.5 \mathrm{~nm}$ laser but we have not observed any Raman signature with this excitation wavelength. To avoid any cross-contamination among different samples via the objective we have used a $\times 50$ long working distance objective (NA: 0.5 ). The theoretical spatial resolution with this configuration is $\approx 0.8 \mu \mathrm{m}$. Power on the sample can be tuned from $10 \mathrm{~mW}$ on a $1.8 \mu \mathrm{m}^{2}$ surface down to $0.1 \%$ of this value using optical filters. Mapping mode was performed on flat zones with steps in $\mathrm{x}$ and y directions equal to $1 \mu \mathrm{m}$. We also used a furnace to perform in-situ Raman measurements from room temperature to $250^{\circ} \mathrm{C}$. Pressure of Ar was kept at 1.5 bar to limit oxidation effect.

Numerical calculations, and in particular DFT, are essential for the interpretation of SERS spectra ${ }^{[16,17]}$. Those calculations have been carried out at the B3PW91/6-31G* level of theory using the GAUSSIANO9 ${ }^{[18]}$ suit of programs. We have calculated the structure of the isolated oxazepam molecule together with its set of harmonic frequencies.

$\underline{\text { Results }}$

\ Oxazepam Gold nanoparticles $\quad 800$ Silica matrix
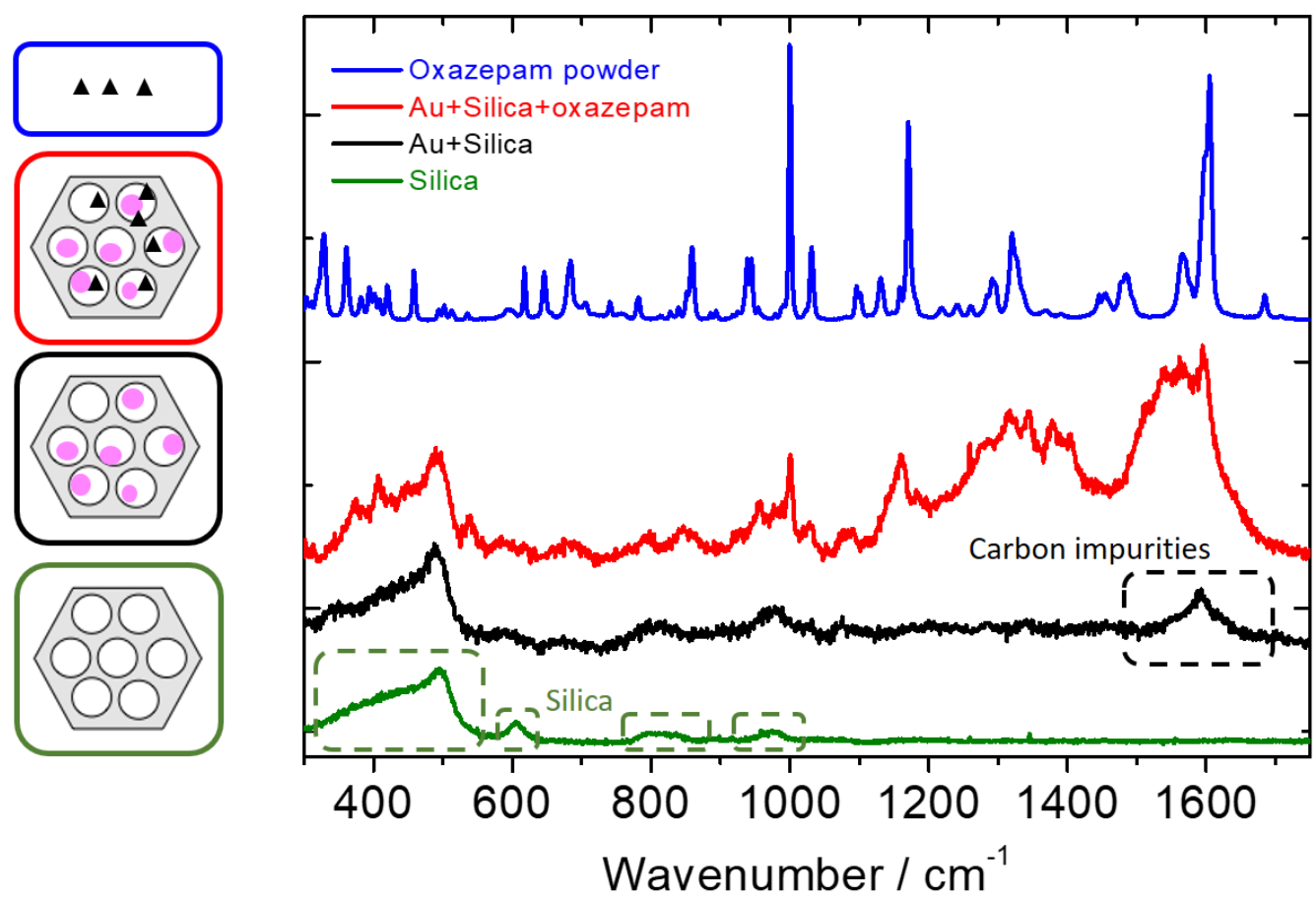

Fig 1: Raman spectra of oxazepam powder (blue, inverted for clarity), oxazepam in silica matrix with gold nanoparticles (SERS, red), silica matrix with gold nanoparticles (black) and silica matrix (green). Excitation wavelength: $633 \mathrm{~nm}$

Typical Raman spectra of the different samples are shown in figure 1. For all of them, the characteristic Raman modes of the silica matrix are clearly observed between 400 and $550 \mathrm{~cm}^{-1}$. Some other weaker modes at around 600,800 and $1000 \mathrm{~cm}^{-1}$ are also associated to the silica matrix ${ }^{[19]}$. When gold nanoparticles are incorporated in the matrix, these modes are still visible, without any significant 
increase of their intensity, suggesting that there is no electromagnetic enhancement between gold and silica. Broad modes at around $1600 \mathrm{~cm}^{-1}$ are visible, they can be associated to carbon impurities formed during the calcination process. When oxazepam molecules are added, the Raman spectrum is strongly modified. It exhibits now the typical features of the SERS modes of this molecule in addition to the modes from the silica matrix. A mode at $270 \mathrm{~cm}^{-1}$ is sometimes observed (see supplementary information). It can be attributed to the Au- $\mathrm{N}$ stretching mode ${ }^{[13]}$ but we do not consider it as a clue for the absorption of oxazepam on gold through its nitrogen atoms as this mode is not systematically observed and it is also present without oxazepam. Its origin remains unclear; we suggest that it could be attributed to the adsorption of nitrogen present in the air.

It has already been reported that the SERS spectrum of oxazepam is not identical to its powder signal, and we clearly observed these differences. This result suggests that we are in experimental conditions where a SERS effect occurs for this molecule with this substrate. To confirm this hypothesis we performed similar measurements with oxazepam adsorbed on the silica matrix without gold nanoparticles and we have not observed any signature from the molecule. This proves the crucial role of gold in the detection of oxazepam.

Gold nanoparticles play two distinct roles in the detection of oxazepam:

-They promote the adsorption of oxazepam molecules in the silica matrix.

-As nano-antenna they enhance the Raman response of the molecules, the so-called Surface Enhanced Raman response.

These two contributions are necessary to get a high sensitivity in the detection of oxazepam molecules, even at low concentration. Usually in SERS, a low concentration induces a low signal to noise ratio. To get a measurable signal, the basic idea is to increase the laser intensity. In figure 2 we report the evolution of the SERS signal with laser power for a fixed concentration.
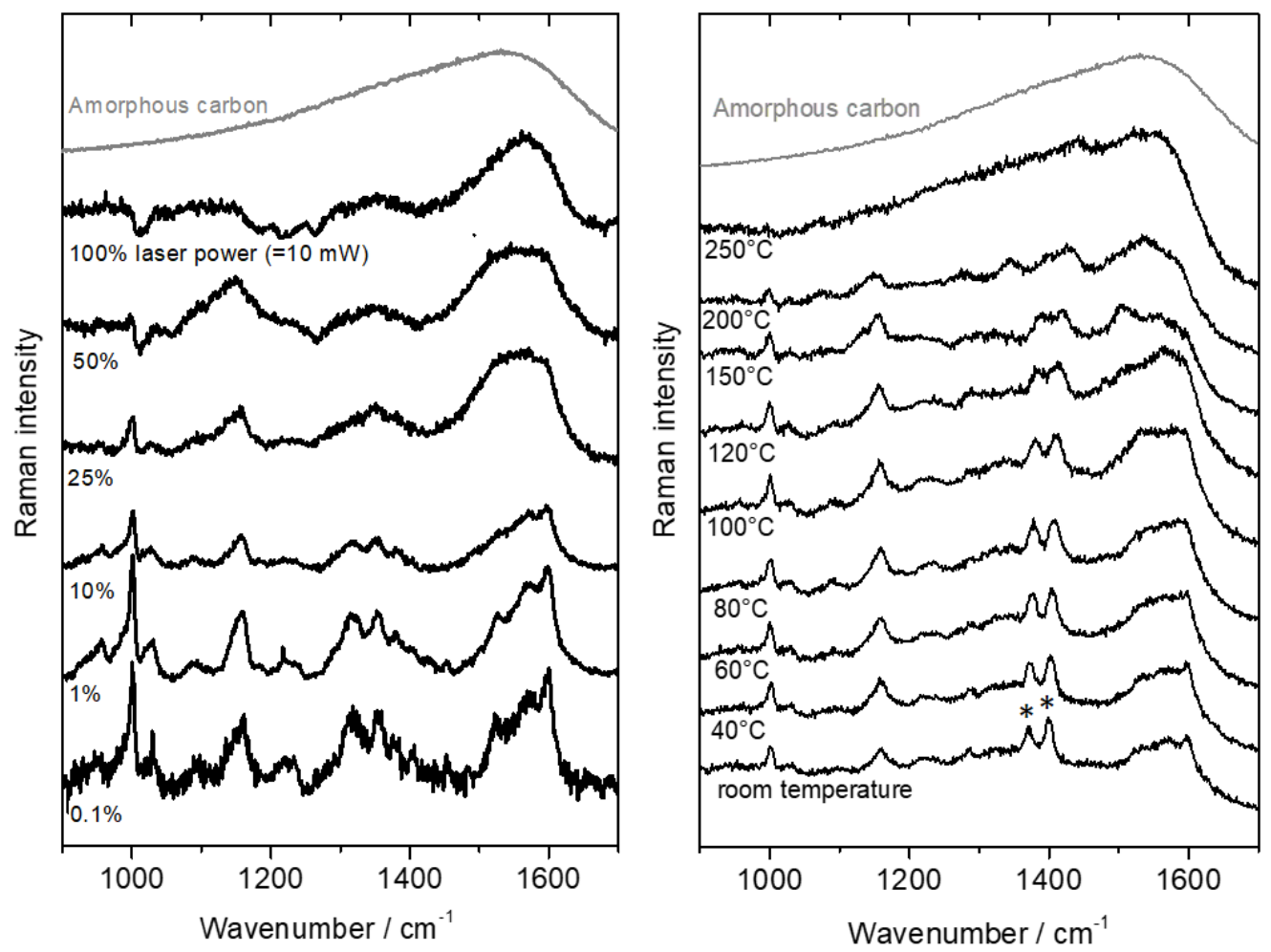
Fig 2: Evolution of the Raman spectrum with laser power (left) and temperature (right). The two peaks marked with a star are coming from the furnace. A typical spectrum of amorphous carbon is also shown for comparison ${ }^{[20]}$. The vertical offset between spectra is only to improve comparability.

We clearly observe an evolution of the SERS spectrum of oxazepam. At low power excitation, all modes are visible and the associated peaks are sharp. They progressively broaden with the increase of laser power and some modes are not visible anymore with laser power higher than $25 \%$. In particular, we note the disappearance of the phenyl band around $1000 \mathrm{~cm}^{-1}$. This first result indicates that laser power plays a role in SERS measurement, it should be maintained to a rather low value (close to 0.5 $\mathrm{mW} / \mu^{2}$ ) as otherwise the recorded spectrum might be modified. For Raman spectroscopy, it is generally admitted that high laser intensity increases the sample temperature and its spectral effect should be similar to high temperature measurement. Thus, in figure 2 we have also reported high temperature measurements up to $250{ }^{\circ} \mathrm{C}$. At first sight, we observe the same features for high temperatures and high laser power: peaks broadening and disappearance of some modes. Nevertheless, if we look carefully at the spectra, some subtle differences are visible. The most obvious is the broadening: it is much more important with temperature increase compared to laser power increase. We can reasonably assume that high temperature degrades the oxazepam molecule. Therefore, the spectral signature of this molecule is progressively transformed into broad $D$ and $G$ bands, characteristic of amorphous carbon. At the maximum tested temperature $\left(250^{\circ} \mathrm{C}\right)$, the SERS spectrum is identical to amorphous carbon confirming the molecule degradation. Such feature has already been observed for $\mathrm{C}_{60}{ }^{[21]}$ for instance. This is clearly not the case with laser power: even if the $G$ band becomes very broad, the $D$ band keeps a low intensity and the final spectrum, at maximum laser power, is different from amorphous carbon. This result suggests that temperature and laser power do not play the same role.

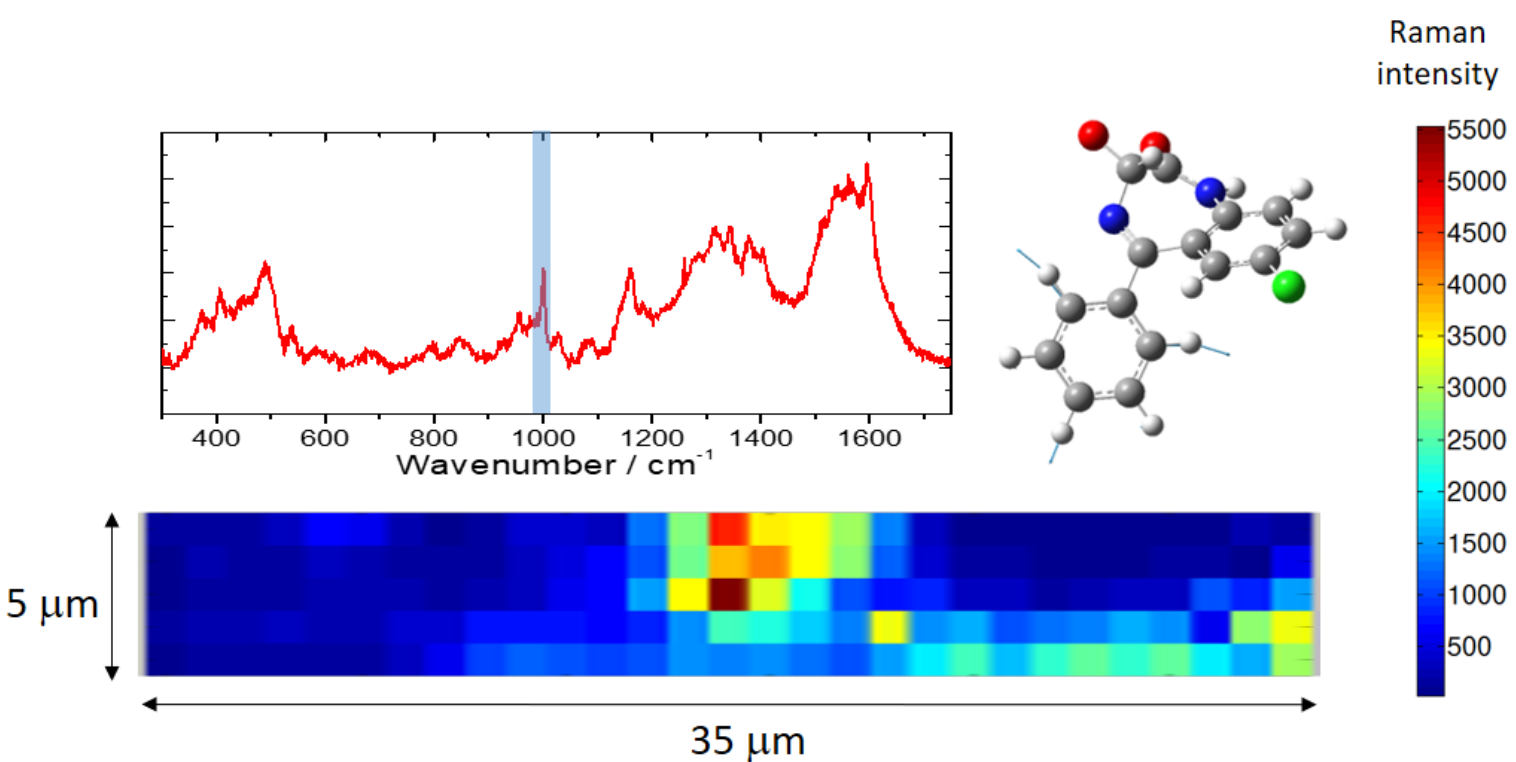

Fig 3: Raman map of the $1000 \mathrm{~cm}^{-1}$ mode of the oxazepam molecule. The size of each pixel is $1 \mu \mathrm{m} \times 1$ $\mu \mathrm{m}$. Excitation wavelength: $633 \mathrm{~nm}$

Another interesting point in our measurements is the spatial inhomogeneity of the SERS signal. In figure 3, a Raman map of the $1000 \mathrm{~cm}^{-1}$ mode of the oxazepam molecule is reported. We focused our attention on this mode for different reasons: it is sharp (easily identifiable), typical of the oxazepam 
molecule (associated to a benzene group vibration as can be seen in figure 3), far from the silica and carbon impurities modes. In addition, high temperature and high laser power measurements have suggested that this mode is sensitive to oxazepam degradation. Thus, it is a clear fingerprint of the oxazepam molecules in the matrix. The mapping exhibits a broad distribution of the mode intensity. Most of pixels exhibit a rather low signal but in some specific places the intensity is greatly enhanced. We can reasonably assume that this result is not related to an inhomogeneous distribution of oxazepam molecules in the silica matrix, even if a slight contribution of this feature cannot be totally excluded. TEM images of the sample (see supplementary information) exhibit a rather homogenous matrix and it is hardly believable that the adsorption of oxazepam molecules should occur in specific places, at least for a micrometer resolution. We rather suggest that this distribution is related to the nano-antenna efficiency. As can be seen in FDTD calculations of the supplementary information, the electromagnetic enhancement depends on the confinement of the gold nanoantenna in the silica pores. We believe that the distribution of Raman intensity shown in figure 3 is related to the distribution of this enhancement. It is clear that a very efficient nano-antenna for the $633 \mathrm{~nm}$ excitation is localized at the pixel in the center of the image giving the highest Raman intensity. This is also probably the reason why we have not observed any signal with the $514.5 \mathrm{~nm}$ excitation, gold nanoantenna being far less efficient tor this excitation wavelength. These features prove once again that we are performing SERS measurements of the oxazepam molecules.

In the map shown in figure 3, we also have an inhomogeneity of the modes distribution. Some typical spectra are visible in figure 4 a). We have focused our attention on two different spectral regions: the first one around $1000 \mathrm{~cm}^{-1}$ and the second at $1600 \mathrm{~cm}^{-1}$.
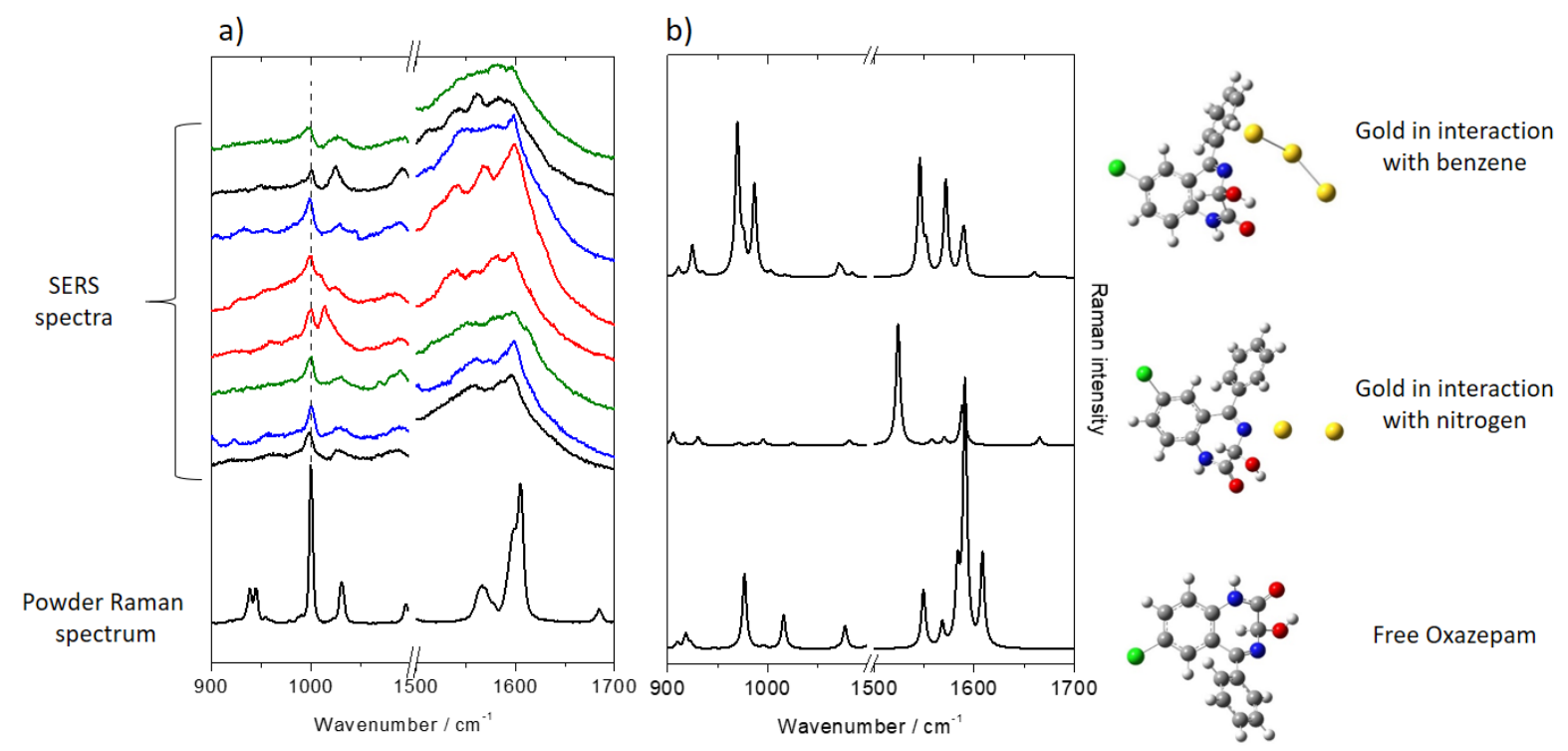

Fig 4: a) Typical experimental SERS spectra of oxazepam extracted from the mapping shown in figure3, in comparison with the powder spectrum. b) DFT calculations of free oxazepam and in interaction with Gold atoms.

It is confirmed here that the SERS spectrum of oxazepam is different from its powder Raman spectrum. We also note that SERS spectra are all slightly different from one location to the other. At $1000 \mathrm{~cm}^{-1}$, the powder Raman spectrum exhibit one intense mode whereas in the SERS spectra a doublet is sometimes observed. In the $1600 \mathrm{~cm}^{-1}$ region, the powder Raman spectrum exhibit two sharp modes whereas SERS spectra show much broader peaks associated to a larger number of modes which cannot be attributed to carbon impurities. Such a feature has already been reported by Trachta et al. ${ }^{[12]}$ : they 
have noticed these differences between SERS and powder Raman spectrum but not the fluctuation in the SERS signal. They attributed those differences to molecular orientation and concluded that the molecule should be adsorbed with the molecular plane orthogonal to the metal surface. As mentioned in the introduction, the role of chemical interaction between the molecule and the metallic surface in essential in SERS ${ }^{[22]}$. For molecules with a strong affinity for gold, such as thiol molecules ${ }^{[23]}$, this question can be easily addressed. This is not the case here: oxazepam has no specific chemical function with a very high affinity for gold. The question of the adsorption mechanism of this molecule remains unclear. This molecule has the possibility to adsorb on the gold surface through different chemical interactions. To check this hypothesis we performed DFT calculations of the molecule in interaction with a few gold atoms. We have not performed the calculation with a complete gold surface as the crystallinity of our gold nanoparticles is certainly poor. The calculated Raman spectra are shown in fig. 4 b). Independently from any molecular orientation effect, it appears clearly that the calculated Raman spectra exhibit spectral differences depending on the position of the gold atoms. When gold atoms are close to the aromatic ring, a doublet appears in the $1000 \mathrm{~cm}^{-1}$ region and 3 bands are present in the $1600 \mathrm{~cm}^{-1}$ region. This result is in good accordance with our measurements. When the gold atoms are located close to the $\mathrm{N}$ atoms, 2 modes are also present in the $1600 \mathrm{~cm}^{-1}$ region, one being shifted down to almost $1500 \mathrm{~cm}^{-1}$. In addition, the calculated spectra do not show modes at $1000 \mathrm{~cm}^{-1}$ anymore. This result suggests that the absence in the SERS spectrum of this mode does not automatically mean that the oxazepam molecule has been degraded. This point is particularly interesting if we recall the influence of laser power in the evolution of the SERS spectrum of oxazepam. We have previously discussed the fact that high temperature transforms the molecule into amorphous carbon but laser power has a different influence: it causes the broadening of the $1600 \mathrm{~cm}^{-1}$ modes, without the appearance of a $D$ band and the $1000 \mathrm{~cm}^{-1}$ mode is not visible anymore (see fig. 2). This is very similar to what is observed in the calculated spectra of oxazepam with gold atoms close to nitrogen, if we take into account the general broadening of Raman modes induced by the inevitable laser heating. Thus we suggest that the evolution of the SERS spectrum of oxazepam is related to changes in adsorption sites of the molecule on the gold surface and not to its degradation. We also propose the same explanation for the spectral fluctuation in the SERS spectrum as shown in fig. 4 . Those fluctuations are the signature of different absorption sites on the gold surface. This hypothesis is corroborated by the comparison between SERS spectra and the powder spectrum. In all SERS spectra, the $1600 \mathrm{~cm}^{-1}$ modes are broader. DFT calculations have demonstrated that many Raman modes are present in the range $1500-1600 \mathrm{~cm}^{-}$ ${ }^{1}$ as soon as the molecule is in interaction with gold atoms. The distribution of various absorption sites on the surface of gold nanoparticles will lead to a general broadening of the modes in the same spectral range, and indeed this is exactly what is experimentally observed. In the $1000 \mathrm{~cm}^{-1}$ spectral range, comparison between measured and calculated spectra leads to the same conclusion: a single intense mode is observed for the pristine molecule, in accordance with powder Raman spectrum, whereas doublets or no modes at all are calculated if the molecule is in interaction with gold, which corresponds exactly to the different measured SERS spectra. We have excellent agreement between calculated and measured spectra considering our hypothesis on the existence of various adsorption sites.

\section{Conclusion}

Based on our DFT calculations and SERS measurements, we can conclude that the differences between powder and SERS spectra of oxazepam are not related to any orientation effect but to specific interactions between the molecule and the gold surface, temperature-dependent adsorption sites to be specific. Any change in the absorption site will cause the observed fluctuations in the SERS spectrum. Our findings also suggest that this mechanism can be activated through laser illumination. This study gives important conclusions for the further development of SERS in drugs detection: first, 
the SERS spectrum can be rather different compared to the powder spectrum and second, the precise control of laser power is essential.

Acknowledgments: this work was funded by the French research national agency (ANR) through the project DOMTOM (16-CE09-0021-05).

[1] B. Yu, M. Ge, P. Li, Q. Xie, L. Yang, Talanta 2019, 191, 1.

[2] C. Muehlethaler, M. Leona, J. R. Lombardi, Anal. Chem. 2016, 88, 152.

[3] S. Cinta Pinzaru, I. Pavel, N. Leopold, W. Kiefer, J. Raman Spectrosc. 2004, 35, 338.

[4] C. Augusto, F. De Oliveira, M. Tadeu, T. Pacheco, K. Lednev, L. Silveira, J. Raman Spectrosc. 2016, 47, 28.

[5] E. C. Le Ru, M. Meyer, P. G. Etchegoin, J. Phys. Chem. B 2006, 110, 1944.

[6] P. G. Etchegoin, E. C. Le Ru, Principles of surface-enhanced Raman spectroscopy and related plasmonic effects, Elsevier, 2009.

[7] H. J. Johnston, G. Hutchison, F. M. Christensen, S. Peters, S. Hankin, V. Stone, Crit. Rev. Toxicol. 2010, 40, 328.

[8] P. A. Mosier-Boss, Nanomaterials 2017, 7, 142.

[9] E. C. Le Ru, P. G. Etchegoin, MRS Bull. 2013, 38, 631.

[10] J. R. Lombardi, R. L. Birke, J. Chem. Phys.

[11] S. Cinta, T. Iliescu, S. Astilean, L. David, O. Cozar, W. Kiefer, J. Mol. Struct. 1999, 482-483, 685.

[12] G. Trachta, B. Schwarze, G. Brehm, S. Schneider, M. Hennemann, T. Clark, J. Raman Spectrosc. 2004, 35, 368.

[13] S. Cinta, L. David, O. Cozar, Spectrosc. Biol. Mol. New Dir. 1999, 565.

[14] H. V. Bandarenka, K. V. Girel, S. A. Zavatski, A. Panarin, S. N. Terekhov, Materials (Basel). 2018, $11,1$.

[15] D. Fernand, D. Bergé-Lefranc, C. Pardanaud, T. N. T. Phan, A. Merlen, V. Hornebecq, Eur. Phys. J. Spec. Top.

[16] A. Merlen, C. Pardanaud, S. Coussan, C. Panagiotopoulos, O. Grauby, C. M. Ruiz, J. Raman Spectrosc. 2018, 49, 1184.

[17] A. Merlen, M. Chaigneau, S. Coussan, Phys. Chem. Chem. Phys. 2015, 17, 19134.

[18] M. J. Frisch, G. W. Trucks, H. B. Schlegel, G. E. Scuseria, M. A. Robb, J. R. Cheeseman, G. Scalmani, V. Barone, B. Mennucci, G. A. Petersson, H. Nakatsuji, M. Caricato, X. Li, H. P. Hratchian, A. F. Izmaylov, J. Bloino, G. Zheng, J. L. Sonnenberg, M. Hada, M. Ehara, K. Toyota, R. Fukuda, J. Hasegawa, M. Ishida, T. Nakajima, Y. Honda, O. Kitao, H. Nakai, T. Vreven, J. A. Montgomery, J. E. Peralta, F. Ogliaro, M. Bearpark, J. J. Heyd, E. Brothers, K. N. Kudin, V. N. Staroverov, R. Kobayashi, J. Normand, K. Raghavachari, A. Rendell, J. C. Burant, S. S. Iyengar, J. Tomasi, M. Cossi, N. Rega, J. M. Millam, M. Klene, J. E. Knox, J. B. Cross, V. Bakken, C. Adamo, J. Jaramillo, R. Gomperts, R. E. Stratmann, O. Yazyev, A. J. Austin, R. Cammi, C. Pomelli, J. W. Ochterski, R. L. Martin, K. Morokuma, V. G. Zakrzewski, G. A. Voth, P. Salvador, J. J. 
Dannenberg, S. Dapprich, A. D. Daniels, Ö. Farkas, J. B. Foresman, J. V. Ortiz, J. Cioslowski, D. J. Fox, 2009, Gaussian, Inc., Wallingford CT.

[19] R. K. Biswas, P. Khan, S. Mukherjee, A. K. Mukhopadhyay, J. Ghosh, K. Muraleedharan, J. Non. Cryst. Solids 2018, 488, 1.

[20] C. Pardanaud, C. Martin, G. Giacometti, N. Mellet, B. Pégourié, P. Roubin, Thin Solid Films 2015, 581, 92.

[21] V. V Brazhkin, A. G. Lyapin, S. V Popova, R. N. Voloshin, Y. V Antonov, Phys. Rev. B 1997, 56, 465.

[22] Y. Fleger, Y. Mastai, M. Rosenbluh, D. H. Dressler, J. Raman Spectrosc. 2009, 40, 1572.

[23] G. Delafosse, A. Merlen, S. Clair, L. Patrone, J. Chem. Phys. 2012, 136, 194704. 